\title{
Development of an Electronic Nose for Olfactory System Modelling using Artificial Neural Network
}

\author{
${ }^{1}$ Mary Anne Roa, ${ }^{2}$ Proceso Fernandez \\ ${ }^{1}$ Department of Information Systems \& Computer Science, Ateneo de Manila University, Philippines; \\ mary.roa@obf.ateneo.edu; pfernandez@ateneo.edu
}

\begin{abstract}
Electronic nose (e-nose) devices have received considerable attention in the field of sensor technology because of their many potential uses such as in identification of toxic wastes, monitoring air quality, examining odors in infected wounds and in inspection of food. Notwithstanding the vast amount of literature on the usage of e-noses for specific purposes, the technology originally and ultimately aims to mimic the capability of mammals to discriminate odors from all sorts of objects. This study demonstrates the theoretical and practical feasibility of designing an e-nose towards general odor classification. A multisensor array hardware unit was carefully constructed for data collection and odor detection. Important hardware design considerations such as sensor calibration, aeration, circuit protection, and voltage/current requirements were satisfied. A highly fine-tuned artificial neural network (ANN) was integrated to the hardware to interpret and relate the data to a target odor class from a set of 10 primary odors identified in a previous study. Various network architecture considerations, such as neuron count, number of layers and activation function, as well as various data treatment methods, such as normalization, and data partitioning, were investigated. The results showed that careful hardware integration with an ANN having sufficiently deep internal structure can yield accurate classification to at least half of the ten primary odor classes, namely fragrant (96\%), fruity (98\%), chemical (99\%), peppermint (98\%), and popcorn (90\%). The results demonstrate the feasibility of making e-noses for general odor classification, which could lead to further broadening of e-nose applications.
\end{abstract}

Keywords: Artificial Neural Network; Odor Classification; Electronic Nose; Machine Learning.

\section{Introduction}

Did you ever measure a smell? Can you tell whether one smell is just twice strong as another? Can you measure the difference between two kinds of smell and another? It is very obvious that we have very many different kinds of smells, all the way from the odor of violets and roses. But until you can measure their likeness and differences, you can have no science of odor. If you are ambitious to find a new science, measure a smell. - Alexander Graham Bell, 1914

The research is supported by the Department of Science and Technology - Engineering Research and Development for Technology, Philippines.

Scientists as well as philosophers have long neglected olfaction. The lack of interest in the nature of smells stems perhaps from its very character. As the most volatile sense of all, the smell does not appear to be 
sufficiently real. Odors are considered as too insubstantial and very brief in appearance [1]. It thus does not come as a surprise that there is no complete understanding of how smell perception works yet, especially in humans [2]. The mechanism of the olfactory system has been studied but not as thoroughly as visual and auditory systems where excellent electrical analogues are available [3]. One of the initial hopes for work in this area was connecting distinct biological attributes with hardware, as well as capturing odor fingerprint, therefore creating an electronic nose (e-nose) [4].

Artificial olfaction can trace its beginnings with the invention of the first gas multi-sensor array in 1982 [5]. Recently, it has received considerable attention largely due to the discovery of numerous applications in diverse fields of applied sciences such as agricultural, biomedical, cosmetics, environmental, food, manufacturing, military, pharmaceutical, regulatory, and more. These include analysis of fuel mixtures [6], detection of oil leaks [7], identification of household odors [8], examining breath [9], analysis of body fluids $[10,11]$ etc. Due to these numerous applications and promising benefits to a diverse field, the global e-nose market has been forecasted to grow by $2.07 \%$ during the period $2016-2020$ [12].

The technology of artificial olfactory system has continuously been advancing up to the present. However, although many e-nose models have been developed, the vast majority of these are intended for very specific applications. Making e-noses adept in categorizing smells of various samples is a big step towards broadening its application.

This study focused on the development of an e-nose that is directed towards general odor classification, by using the 10 primary smell classes suggested by researchers from University of Pittsburgh and Bates College [13]. Their study presented that odor dimensions apply categorically and that scents can be placed in one of ten basic categories of odor -- fragrant, woody, fruity, chemical, peppermint, sweet, popcorn, lemon, pungent, and decayed.

This study integrated different olfaction sensors in a compact e-nose prototype that can make fine discrimination on the chemical properties of odors from different objects. An Artificial Neural Network (ANN) was used to make reasonable decisions about the categories of the smell. Various ANN configurations and data treatment methods affecting overall system performance were investigated.

\section{Methodology}

\subsection{Hardware Design Consideration}

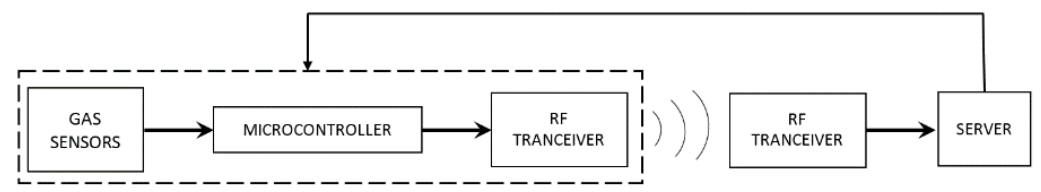

Figure 1 Overall System Block Diagram

Hardware design is the elaborate process to make a prototype to satisfy requirements. The process of designing e-nose generally begins with fulfilling the need for air to flow into the assembly to remove various types of gaseous and particulate contaminants that may affect or interfere with measurements. The sensor elements' calibration is equally vital. To design the auxiliary circuit, current specification, voltage specification and transient protections were considered to meet the specific functional requirements on power regulation. Figure I show the overall system block diagram. 


\subsubsection{Sensor Integration}

An e-nose fundamentally consists of three major parts, and these are the sample delivery system, detecting system, and processing system. Detection typically uses an array of sensors of different sensitivities that simultaneously respond to the volatile chemicals present in a sample. The pattern of response for different odorants is important, as this distinguishability allows the system to identify an odor. In this study, the prototype was realized by interfacing 10 semiconductor gas sensors, namely MQ2 for smoke, MQ3 for alcohol, MQ4 for natural gas, MQ5 for LPG, MQ6 for butane and propane, MQ7 for carbon monoxide, MQ8 for hydrogen gas, MQ9 for methane, MQ135 for benzene and MQ138 for ammonia. The sensors send measurements to the server through a ZigBee transmitter. The configuration of the MQ gas sensor is shown in Figure II.

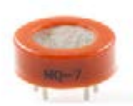

(a)

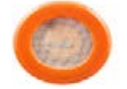

(b)

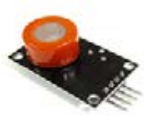

(c)

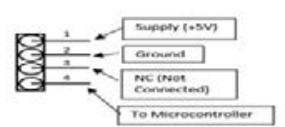

(d)

Figure 2. Sensor (a) front view, (b) top view, (c) module and (c) pin configuration

\subsubsection{Aeration}

The sample delivery system is essential to guarantee stable operating conditions and to avoid contamination of samples from external odor sources. Insulation or sealing materials that may give off fumes such as silicone sealants and solvent-based adhesives were avoided. Gas is supplied to detectors using a static gas mixture, thus avoiding direct gas flow onto the sensor that can cause a false high reading. Ambient air can pass into the sampling chamber after every gas exposure cycle using an axial flow fan that draws air into the case from the outside and expels a previous sample's gases from the inside.

\subsubsection{Auxiliary Requirements:}

A schematic diagram of the power supply used is shown in Figure III. A voltage regulator maintains the sensor output voltage level requirement of 5 VDC at point (a). Another integrated circuit at point (b) was used to further regulate the output to $3.3 \mathrm{~V}$ in order to satisfy the requirements of the ZigBee module and the microcontroller. The microcontroller used, described in Figure IV, serves as the controller for the different sensors and auxiliary components of the system.

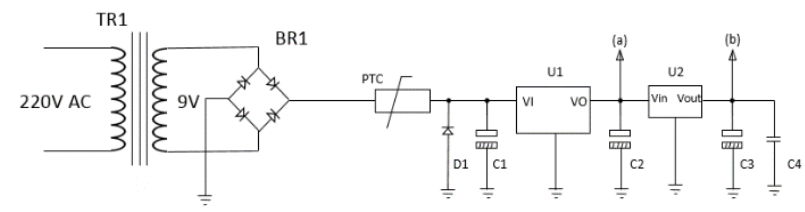

Figure 3. Main Supply Schematic Diagram

Figure $\mathrm{V}$ depicts a scale model of the assembly. The bigger block serves as the sample delivery chamber where an object being analyzed is placed and into which odorless air is ducted after sampling. The adjacent smaller block houses the auxiliary electronics. 


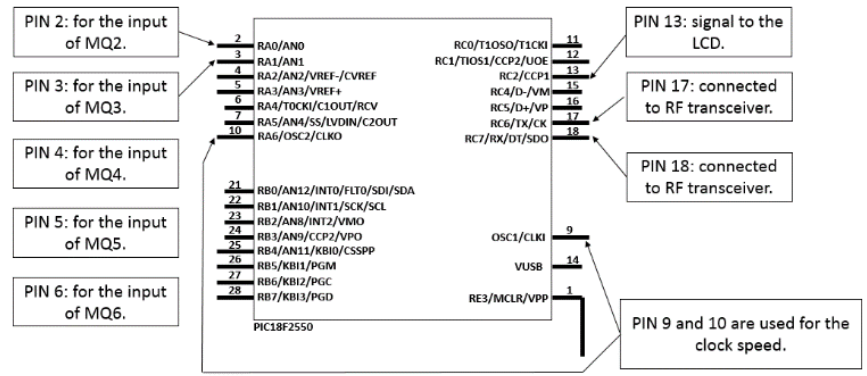

Figure 4. Microcontroller Unit Connection Diagram

The output category of smell is displayed on an LCD monitor integrated to the e-nose prototype. Options to "classify" and "clear" allow a user to trigger the device to either read and process samples or turn the exhaust for clearing gas out of the chamber.
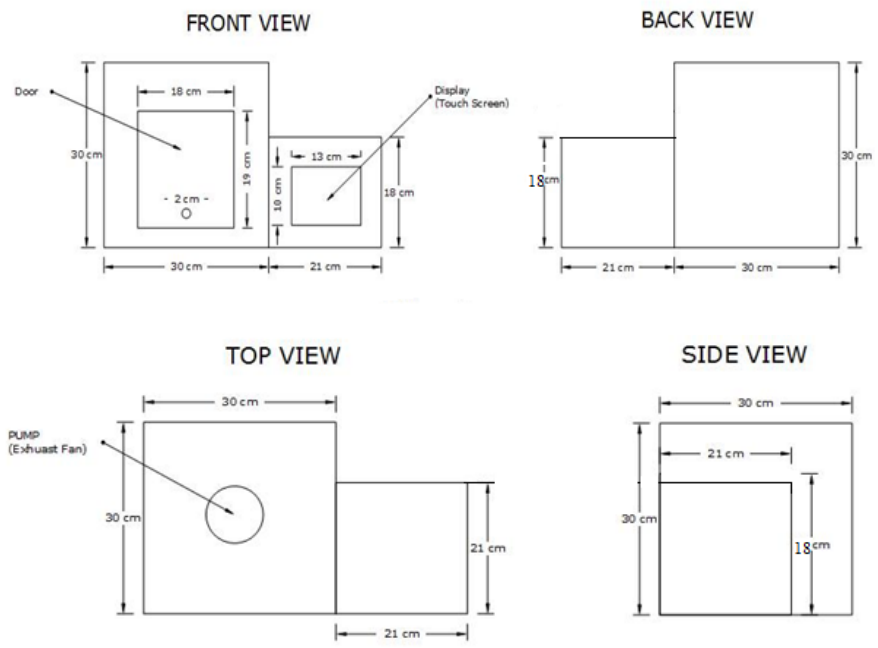

Figure 5. Orthographic Projection of the Prototype

\subsection{Software Configuration}

An artificial neural network (ANN) interprets the multi-dimensional data yield by the hardware unit and relates this to a target odor class. The ability of an ANN to accurately classify the odor sample depends a lot on both its model parameters, i.e., network connection weights, and on its hyperparameters that include the number of layers, the number of neurons in each layer and the activation function used in a neuron. The network parameters are typically set through training the network using some form of gradient descent learning algorithm combined with back propagation. The hyperparameters, on the other hand, are typically handcrafted. To achieve good accuracy, extensive experimentation was conducted on various ANN hyperparameters, as discussed in the succeeding paragraphs. Figure VI hints on the overall iterative nature in the design of the final ANN used in the e-nose for this study. 

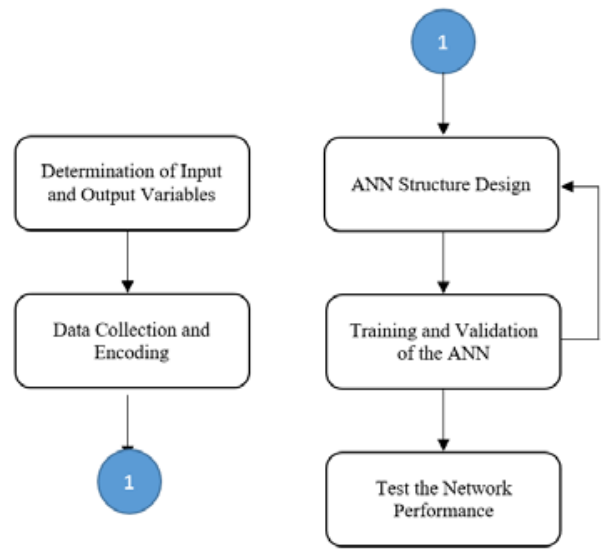

Figure 6. Artificial Neural Network Design Workflow

\subsubsection{Neuron Count}

The neurons in the hidden layer are responsible for the internal representation of the data and the information transformation between input and output layers. A preliminary study [14] on the effect of neuron count on odor classification showed that small networks were unable to model the odor pattern. An increase in the number of neurons in the hidden layers resulted in better fit. However, increasing the number of neurons beyond some threshold has an opposite effect when verified against the test data. This is because having more hidden neurons means that there are a lot more model parameter to be learned, and the corresponding much larger (optimization) search space makes it more difficult to find the optimal parameters.

\subsubsection{Number of Layers}

As ANNs can consist of multiple layers of computational units, various network structures were also trained and tested in order to find out the relationship between the number of hidden layers against the overall classification performance. Results from a previous work [15] showed that the structure of the network has a perceptible impact on learning time. Evidently, large networks took longer time to learn the characteristics of the data. However, an ANN with small internal structure did not give good approximations even for patterns included in its training set. Increasing the number of layers improved generalization of the network. In this paper, we denote the network topology by a sequence of $m$ numbers that describe the sizes of the $m$ layers. For instance, 10-3-9-10 describes a network with 10 neurons in the first layer, 3 in the second layer, 9 in the third layer, and 10 neurons in the last layer.

\subsubsection{Activation Function}

The activation function controls the total signal that a neuron produces given its input. It affects the power of a neural network in several aspects such as efficiency of weight updates, the speed of learning and final network complexity. Quantitative comparisons of four of the most commonly used ANN activation functions were explored in [16]. Networks with linear activation functions are consistently fastest, followed closely by those with Rectified Linear Unit (ReLU). The sigmoid and hyperbolic tangent activation functions involve more costly computations and are thus significantly slower, but they generally yield the most accurate results. However, the difference in the accuracies between the ReLU and the sigmoid or 
hyperbolic tangent is generally not significant when a properly trained network has sufficient number of hidden layers and neurons in each layer. The faster learning capability with the use of ReLU, when compared with the other non-linear activation functions, thus makes it the preferable activation function for large models trained on large datasets.

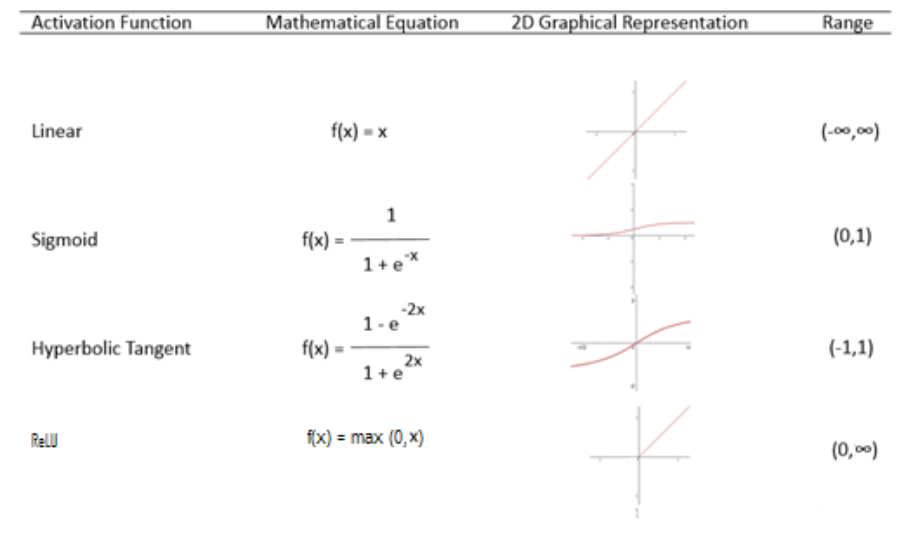

Table 1. Activation Functions

\subsection{Data Collection and Treatment}

A big challenge in e-nose design, besides hardware and software design considerations, is getting and processing the right data. ANN design is generally better when the number of training samples is substantial, as this allows for more free parameters to be incorporated in the model. The sample data collection was done carefully by ensuring the proper working conditions of the e-nose hardware unit. Any traces of smoke, adhesives, gases, or solvents were removed from the chamber, and the atmospheric conditions in the chamber were maintained stable at room temperature and humidity.

A series of measurements was captured for each sample. Each measurement comprised of an exposure cycle followed by a cleaning phase where the ambient air was passed into the chamber through the axial flow fan. The exposure was fixed at 15 seconds to provide enough time for a quantifiable response from all the sensors. The cleaning phase was set to 15 seconds to permit an acceptable recovery of the sensors sensitive to the sampled material and ensure that all gases or fumes are expelled from inside the chamber.

During the sample data collection, five measurements for each sensor were obtained and sent to the server for processing. This was repeated 3 times per sample. Thus, a raw data matrix with dimension $15 \times 10$ for each sample was obtained. The 10 sensor measurements, 15 collected readings per sample, and 100 different sample materials collectively yielded a 1500×10 (readings by feature/sensor) dataset that can be downloaded from [17]. The dataset is balanced, with each odor class having 150 sample readings based on 10 different sample materials per odor class.

The collected dataset was used in different ways to benchmark candidate ANNs under various experiment configurations. Specifically, the dataset was used in investigating the effect on the ANN performance of data pretreatment through normalization, in exploring for a good partitioning of the normalized dataset, and then in searching for the ANN hyperparameters and parameters using the 10-fold validation results as estimates of ANN performance. 
Mary Anne Roa, Proceso Fernandez; Development of an Electronic Nose for Olfactory System Modelling using Artificial Neural Network. Transactions on Machine Learning and Artificial Intelligence, Volume 6 No 4 August (2018); pp: 30-41

\subsubsection{Data Normalization}

Working directly on raw data with arbitrary units and different magnitudes could affect the learning algorithm negatively because the features with bigger values may dominate those with smaller ones. Normalization of the input data is usually carried out in order to address this issue.

In this study, the hyperparameter search was performed on raw and normalized data (min-max and zscore). The results, in terms of precision (P), recall (R) and f-measure (f), are summarized in Table II and clearly indicate the benefit of performing data normalization. Aside from producing better scores, training the ANN on normalized data, also allowed relatively smaller networks to achieve accurate classification.

Table 2. Hyperparameter Tuning Results with Normalization

\begin{tabular}{|c|c|c|c|c|}
\hline Data Treatment & ANN & $\mathrm{P}$ & $\mathrm{R}$ & $\mathrm{f}$ \\
\hline \multirow{4}{*}{ Raw } & $10-10-6-8-10$ & 0.61 & 0.61 & 0.60 \\
\cline { 2 - 5 } & $10-8-6-10-10$ & 0.68 & 0.68 & 0.67 \\
\cline { 2 - 5 } & $10-8-4-8-10$ & 0.74 & 0.76 & 0.73 \\
\cline { 2 - 5 } & $10-9-10-10$ & 0.76 & 0.78 & 0.77 \\
\hline \multirow{3}{*}{ Min-max scaled } & $10-7-2-3-10$ & 0.73 & 0.75 & 0.74 \\
\cline { 2 - 5 } & $10-10-7-10$ & 0.80 & 0.79 & 0.79 \\
\cline { 2 - 5 } & $10-8-5-10$ & 0.83 & 0.82 & 0.82 \\
\cline { 2 - 5 } & $10-3-9-10$ & 0.83 & 0.82 & 0.82 \\
\hline \multirow{3}{*}{ Z-standardized } & $10-5-4-3-10$ & 0.72 & 0.72 & 0.72 \\
\cline { 2 - 5 } & $10-6-3-5-10$ & 0.74 & 0.76 & 0.75 \\
\cline { 2 - 5 } & $10-9-8-10$ & 0.79 & 0.80 & 0.79 \\
\cline { 2 - 5 } & $10-8-9-10$ & 0.84 & 0.84 & 0.84 \\
\hline
\end{tabular}

\subsubsection{Dataset Partitioning}

A general practice for improving generalization when training an ANN is to first divide the available data into three subsets -- training, validation and test sets. This partitioning is called the holdout method. To investigate the impact on the ANN model performance of the proportion of data in the holdout method, ANN models from hyperparameter tuning were trained on a partitioned min-max normalized dataset, and errors on the test subsets were observed. Results in Table III show that the best overall result was obtained when $80 \%$ of the data is used for training, $10 \%$ for validation and $10 \%$ for testing. It is not a recommended approach to cut the test subset excessively, as in 80-15-5, as it affects negatively the generalizing power of an ANN. On the other hand, leaving no data for validation, as in 90-0-10, makes overfitting much more likely to happen.

Table 3. Result of Holdout Partitioning on Pre-Configured ANN Configurations

\begin{tabular}{|c|c|c|c|}
\hline $\begin{array}{c}\text { Dataset } \\
\text { Partitioning }\end{array}$ & $\begin{array}{c}\text { Training } \\
\text { Accuracy }\end{array}$ & $\begin{array}{c}\text { Validation } \\
\text { Accuracy }\end{array}$ & $\begin{array}{c}\text { Testing } \\
\text { Accuracy }\end{array}$ \\
\hline $70-15-15$ & 0.82 & 0.81 & 0.72 \\
\hline $80-15-5$ & 0.79 & 0.73 & 0.75 \\
\hline $90-0-10$ & 0.77 & N/A & 0.71 \\
\hline $80-10-10$ & 0.87 & 0.81 & 0.89 \\
\hline
\end{tabular}




\subsubsection{K-fold Validation:}

In searching for ANN hyperparameters and parameters, we employed K-fold validation to get very good estimation of the expected performance of each candidate ANN. The value $\mathrm{K}=10$ was chosen, not only because this is the general practice in many other studies, but also because in each of the $\mathrm{K}$ iterations, the dataset can be partitioned into 80-10-10 for training-validation-testing which is consistent to what was earlier observed to be a good partitioning of the dataset.

Furthermore, recall that the dataset consists of 15 readings for each of the 100 different material samples (10 different material samples for each of the 10 odor classes). Data stratification was done in distributing the 10 different samples per odor class to the 10 folds, so that each fold contains samples from all the odor classes. However, while the material samples were distributed evenly, the 15 readings of each material sample were crucially grouped together under the same fold so that these similar readings from the same material in the test subset were not used to train the network, thus ensuring the integrity of test results.

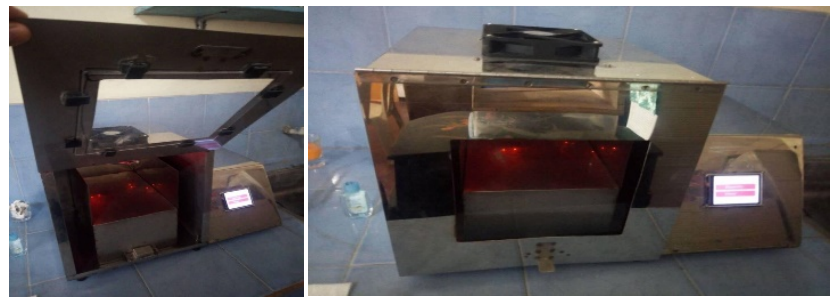

Figure 7. E-nose Prototype

\section{Results and Discussions}

The e-nose prototype is shown in Figure VII. The hyper parameters were tuned via random search to configure an optimum network architecture. The best odor classification performance, in terms of precision, recall, and f-measure, was achieved using a 10-3-9-10 ANN shown in Fig. 8. The ReLU activation function was implemented for the hidden layers of the final ANN because of its fast learning capability while yielding good accuracy. Training was done on a min-max normalized dataset and 10-fold cross-fold validation was implemented to estimate the generalizing power of the different neural network configurations.

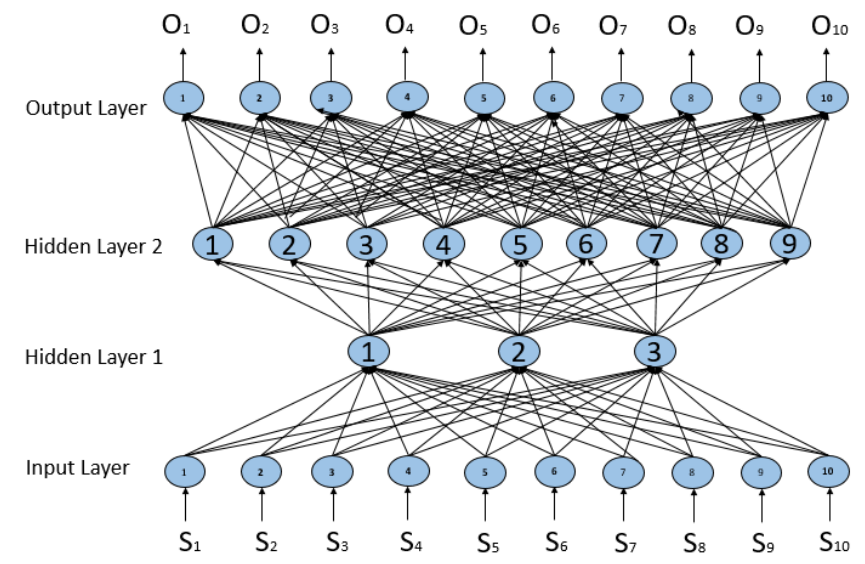

Figure 8. Final ANN Architecture 
Mary Anne Roa, Proceso Fernandez; Development of an Electronic Nose for Olfactory System Modelling using Artificial Neural Network. Transactions on Machine Learning and Artificial Intelligence, Volume 6 No 4 August (2018); pp: $30-41$

The consolidated confusion matrix in Table IV shows very accurate classification to at least half of the 10 classes. Accuracies of over $90 \%$ were obtained for the following 5 odor classes: Fragrant (96\%), Fruity (98\%), Chemical (99\%), Peppermint (98\%) and Popcorn (90\%). In addition, the performance on Woody is within an acceptable value of $82.7 \%$. A closer investigation of the sensor measurements per odor class reveals that the classes with relatively inferior results (decaying, pungent, citrus, sweet) have several sensors with comparatively close measurements. For example, about $30 \%$ of decaying samples were incorrectly classified as citrus, and the sensor readings for MQ3, MQ7, MQ8, MQ135, MQ138 were within very close ranges.

Table 4. Final ANN Confusion Matrix

\begin{tabular}{|c|c|c|c|c|c|c|c|c|c|c|}
\hline Class & Fragrant & Woody & Fruity & Chemical & Minty & Decaying & Pungent & Citrus & Popcorn & Sweet \\
\hline 1 & 144 & 5 & 1 & 0 & 0 & 0 & 0 & 0 & 0 & 0 \\
\hline 2 & 13 & 124 & 1 & 0 & 12 & 0 & 0 & 0 & 0 & 0 \\
\hline 3 & 2 & 0 & 147 & 1 & 0 & 0 & 0 & 0 & 0 & 0 \\
\hline 4 & 0 & 1 & 0 & 149 & 0 & 0 & 0 & 0 & 0 & 0 \\
\hline 5 & 0 & 0 & 0 & 0 & 148 & 0 & 2 & 0 & 0 & 0 \\
\hline 6 & 0 & 0 & 0 & 0 & 0 & 71 & 6 & 44 & 17 & 12 \\
\hline 7 & 0 & 0 & 0 & 0 & 28 & 4 & 83 & 0 & 24 & 11 \\
\hline 8 & 0 & 28 & 1 & 0 & 2 & 17 & 0 & 102 & 0 & 0 \\
\hline 9 & 0 & 0 & 0 & 0 & 0 & 0 & 1 & 0 & 136 & 13 \\
\hline 10 & 0 & 0 & 0 & 0 & 0 & 4 & 28 & 0 & 30 & 88 \\
\hline
\end{tabular}

The MQ-series gas sensors have partial sensitivity and they can respond marginally to a range of gases. A boxplot of each sensor for each odor class in Fig. 9 reveals the overlaps between the ranges of measurements on different scents, making the classes harder to set apart. For this reason, more sophisticated sensors may be employed for future studies. Moreover, boxplots for Sensor 9 (MQ135) and Sensor 6 (MQ7) are predominantly similar. It seems that removing one of these sensors is not likely to impact the e-nose performance negatively.

Table 5. Class Precision, Recall and F-Measure for the different Odor Classes

\begin{tabular}{|c|c|c|c|}
\hline Class & Precision & Recall & F-measure \\
\hline Fragrant & 0.960000000 & 0.905660377 & 0.932038835 \\
\hline Woody & 0.826666667 & 0.784810127 & 0.805194805 \\
\hline Fruity & 0.980000000 & 0.980000000 & 0.980000000 \\
\hline Chemical & 0.993333333 & 0.993333333 & 0.993333333 \\
\hline Peppermint & 0.986666667 & 0.778947368 & 0.870588235 \\
\hline Decaying & 0.473333333 & 0.739583333 & 0.577235772 \\
\hline Pungent & 0.553333333 & 0.691666667 & 0.614814815 \\
\hline Citrus & 0.680000000 & 0.698630137 & 0.689189189 \\
\hline Popcorn & 0.906666667 & 0.657004831 & 0.761904762 \\
\hline Sweet & 0.586666667 & 0.709677419 & 0.642335766 \\
\hline
\end{tabular}


Additionally, the confusion matrix in Table IV shows that classes with inferior results (decaying, pungent, citrus, sweet) have a good number of samples misclassified as belonging to one of the other classes. For instance, $9 \%$ false of the decaying samples were misclassified as citrus and $11 \%$ of the citrus samples were misclassified as decaying. Using a second pass two-odor ANN classifier chained to the original 10-class ANN network, in a future study, may help address some of these error-prone classifications.
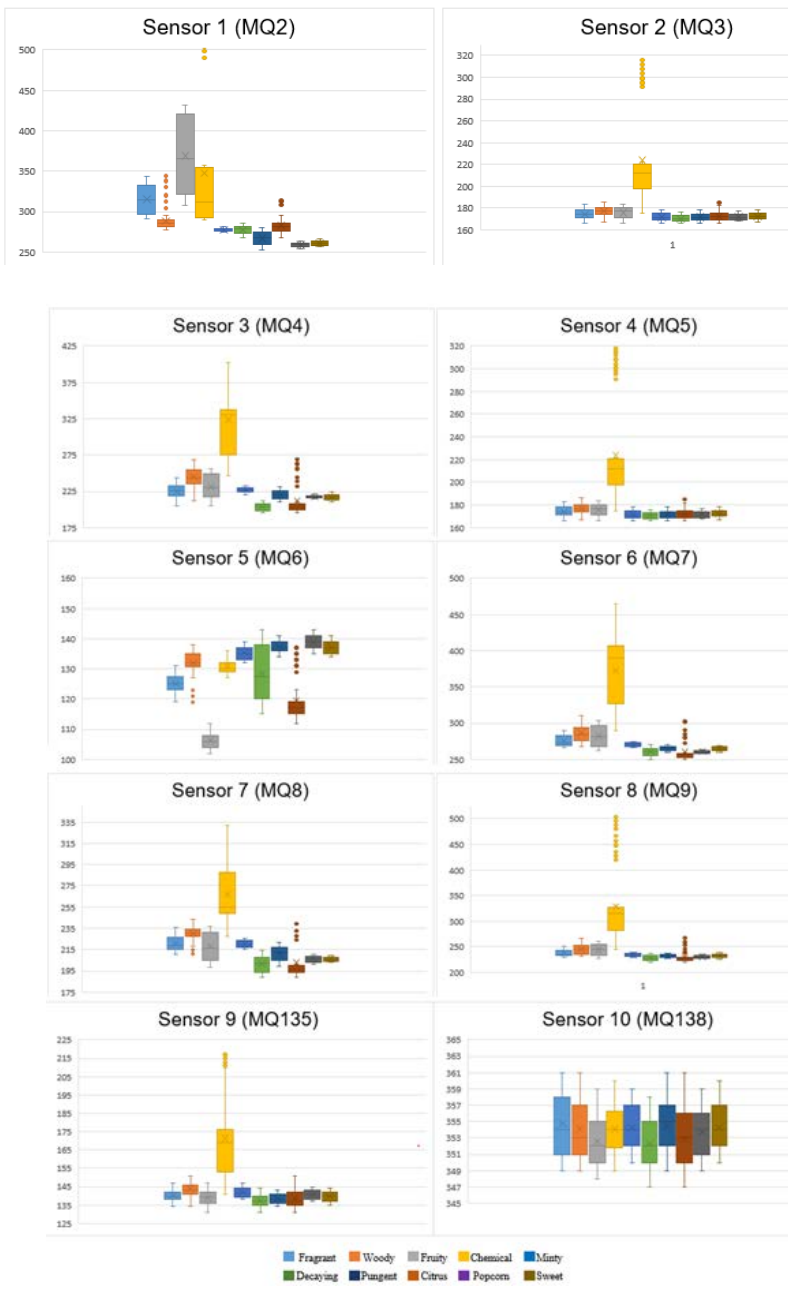

Figure 9. Measurement Box Plots per Sensor

\section{Conclusion}

This study presented the feasibility of making an e-nose prototype intended for general odor classification. The system was realized by integration of hardware unit, which yields multi-dimensional odor measurement data for each sample, with an ANN that interprets and relates the data to a target class. The hardware unit is mainly comprised of an array of ten gas sensors, a microprocessor which serves as the data acquisition and interfacing component, a supply system for power requirements, a protection circuitry against voltage and current transients, an exhaust system to facilitate removal of gas and generate inward flow of air, and a compact chamber designed to allow easy sampling. The prototype served to provide a working dataset for ANN training purposes and to test the performance of the end design e-nose system. The final e-nose classifier with topology 10-3-9-10 and ReLU activation function returns very accurate predictions to at least half of the classes, namely fragrant (96\%), fruity (98\%), chemical (99\%). peppermint (98\%) and popcorn (90\%). 
Mary Anne Roa, Proceso Fernandez; Development of an Electronic Nose for Olfactory System Modelling using

Artificial Neural Network. Transactions on Machine Learning and Artificial Intelligence, Volume 6 No 4 August (2018); pp: 30-41

Future studies may investigate improving the generalization power of the ANN by exploring other types of ANN architectures, utilizing more sophisticated hardware sensors, and even augmenting on the gathered data to allow for better ANN training.

\section{Acknowledgment}

The research is supported by the Department of Science and Technology - Engineering Research and Development for Technology, Philippines. The authors wish to express deep gratitude to Dr. Rosula Reyes, Dr. William Yu, Dr. John Paul Vergara and Dr. Ariel Maguyon for their valuable inputs, motivation and support in this study.

\section{REFERENCES}

[1] Barwich, "A sense so rare: measuring olfactory experiences and making a case for a process perspective on sensory perception." Biol Theory 9:258-268, 2014.

[2] L. Harman, "Human relationship with fragrance," In: The chemistry of fragrances: from perfumer to consumer. Royal Society of Chemistry, Cambridge, 2006, pp 1-2.

[3] D. Schild and J.W. Gardner, "Detection and coding of chemical signals: a omparison between artificial and biological systems," University of Warwick, 1991.

[4] N. Barsan, and U. Weimar, "Electronic nose: current status and future trends," Institute of Physical and Theoretical Chemistry, University of Tubingen, Germany, 2008.

[5] K. Persaud, and G. Dodd, "Analysis of discrimination mechanisms in the mammalian olfactory system using a model nose," Nature. 299:352-355, 1982.

[6] P.E. Keller, R.T. Kouzes, and L.J. Kangas, "Three neural network based sensor systems for environmental monitoring," IEEE Electro 94 Conference Proceedings, Boston, MA, 1994, pp.377-382.

[7] R.J. Lauf and B.S. Hoffheins, "Analysis of liquid fuels using a gas sensor array," Fuel , vol. 70, 1991, pp. 935940.

[8] H.V. Shurmur, "The fifth sense: on the scent of the electronic nose," IEEE Review, March 1990, pp. 95-58.

[9] Amico, A. Natale, C. Paolesse, and R. Macagnano, "Olfactory systems for medical applications" Sens. Actuators B Chem., 1, 2008, 458-465.

[10] I.A.Casalinuovo, D. di Pierro, M. Coletta, P. di Francesco, "Application electronic noses for disease diagnosis and food spoilage detection," Sensors, 6, 2008, 1428-1439.

[11] H. Sun, F. Tian and Z. Liang, "Sensor array optimization of electronic nose for detection of bacteria in wound infection," IEEE Transactions on Industrial Electronics, Volume 64, Issue 9, 2017.

[12] Norah Trent, “ Global Electronic Nose Industry 2016 Market Research Report, “ 2016. 
[13] J. Castro, A. Ramanathan, and C. Chennubhotla, "Categorical dimensions of human odor descriptor space revealed by non-negative matrix factorization," 2013.

[14] M. Roa and P. Fernandez, "A study of the effect of network architecture in artificial neural network performance applied to electronic olfactory device, National Graduate Student Leadership and Research Conference, Laguna, Philippines, 18-19 August 2016.

[15] M. Roa and P. Fernandez, "Study on optimization of artificial neural network generalization power based on architecture," Proceedings of 90th The IIER International Conference, Dubai, UAE, 1st-2nd January 2017, ISBN: 978-93-86291-78-3.

[16] M. Roa and P. Fernandez, "An empirical study of different artificial neural network activation functions applied to five classification problems," submitted, 2017.

[17] M. Roa and P. Fernandez, "Development of an Electronic Nose for Olfactory System Modelling using Artificial Neural Network," 2018. [Online]. Available: https://www.researchgate.net/project/Development-of-an-Electronic-Nose-for-Olfactory-SystemModelling-using-Artificial-Neural-Network

[18] S. Glen, "Alpha Level (Significance Level): What is it? Retrieved," $2012 . \quad$ from: http://www.statisticshowto.com/what-is-an-alpha-level/ 